\title{
La resolución de problemas y el desarrollo del pensamiento matemático
}

\author{
Problem solving and the development of mathematical thinking
}

Resolução de problemas e desenvolvimento do pensamento matemático

ARTÍCULO DE INVESTIGACIÓN

\author{
Wilver Vargas Rojas \\ wilvervargas11@gmail.com \\ ORCID: 0000-0001-9177-0032
}

Universidad Autónoma del Beni-ALSIE consultores, Bolivia

Recibido 20 de enero 2021 | Arbitrado y aceptado 05 de marzo 2021 | Publicado en marzo 2021

\section{RESUMEN}

La resolución de problemas y el desarrollo del pensamiento lógico erróneamente son concebidos y abordados de manera causal. Siendo que al caracterizar la enseñanza aprendizaje de la Matemática en una institución escolar se evidencia que los estudiantes no resuelven problemas lógico matemático. Frente a ello, se propone una metodología de enseñanza aprendizaje de la Matemática orientada al desarrollo del pensamiento lógico matemático. La investigación se valió de la observación científica, el procesamiento dialéctico y el deductivo de la información, para finalmente arribar a una propuesta centrada en el aprendizaje desarrollador y sus dimensiones activación - regulación, significatividad y motivación, conjuncionado con las seis etapas o momentos de la función instructiva del proceso de enseñanza aprendizaje que son la esencia de la propuesta metodológica, integrando de esta manera la instrucción y el desarrollo del pensamiento lógico Matemático.

Palabras clave: Pensamiento lógico; resolución de problemas

\section{ABSTRACT}

Problem solving and the development of logical thinking are erroneously conceived and approached causally. Being that when characterizing the teaching learning of Mathematics in a school institution, it is evident that students do not solve mathematical logical problems. Faced with this, a teaching-learning methodology of Mathematics is proposed aimed at the development of mathematical logical thinking. The research made use of scientific observation, dialectical and deductive processing of information, to finally arrive at a proposal focused on developer learning and its activation regulation, significance and motivation dimensions, combined with the six stages or moments of the instructive function of the teaching-learning process that are the essence of the methodological proposal, thus integrating instruction and the development of logical mathematical thinking.

Key words: Logical thinking; problem resolution 


\section{RESUMO}

A resolução de problemas e o desenvolvimento do pensamento lógico são erroneamente concebidos e abordados causalmente. Sendo que ao caracterizar o ensinoaprendizagem de Matemática em uma instituição escolar, fica evidente que os alunos não resolvem problemas matemáticos lógicos. Diante disso, é proposta uma metodologia de ensinoaprendizagem da Matemática voltada para o desenvolvimento do pensamento lógico matemático. A pesquisa valeu-se da observação científica, do processamento dialético e dedutivo da informação, para finalmente chegar a uma proposta voltada para a aprendizagem do desenvolvedor e sua ativação - dimensões regulação, significância e motivação, combinadas com as seis etapas ou momentos da função instrutiva do ensino -processos de aprendizagem que são a essência da proposta metodológica, integrando assim o ensino e o desenvolvimento do pensamento lógico-matemático.

Palavras-chave: Pensamento lógico; resolução de problemas

\section{INTRODUCCIÓN}

La resolución de problemas es una situación de aprendizaje que ha sido adoptado casi de manera generalizada a partir de autores como Borasi (1986), "desde el punto de vista epistemológico el problema es la situación que muestra un determinado objeto, que genera en un sujeto la necesidad de su modificación" (Álvarez, 2004, p. 46). Consiste en presentar situaciones problematizadas donde existe un grado de dificultad para resolverlos, eso determina si se trata de un problema o un ejercicio, ya que el problema no tiene, a primera vista para el sujeto, una resolución asequible.

Diferentes investigadores han resaltado el papel de la resolución de problemas en el proceso de enseñanza aprendizaje de la Matemática. Entre los estudios más sobresalientes se tiene los trabajos de Polya en Hungría y Estados Unidos (desarrolló el método denominado el "Método de Polya" para resolver problemas de manera sistemática), Miguel de Guzmán en España (propone una serie de métodos deductivos e inductivos para resolver problemas), Santos Trigo en México (estudia la resolución de problemas y plantea que se trata de una actividad esencial de aprendizaje), Schoenfeld de la Universidad de Berkeley en Estados Unidos (investiga la resolución de problemas llegando a caracterizar los diferentes procesos que se desarrollan bajo este denominativo), Llivina y Jiménez (plantean un enfoque orientado hacia el proceso de enseñanza-aprendizaje de la resolución de problemas matemáticos), Castellanos (desarrolla el concepto de enseñanza desarrolladora y sus dimensiones: activaciónregulación, significatividad y motivación) y Ron Galindo en Cuba (desarrolla una estrategia metodológica a partir de las dimensiones propuestos por Castellanos)..

En el contexto boliviano, la resolución de problemas ha sido adoptado como elemento metodológico para la enseñanza de la Matemática, de manera oficial, a partir de la promulgación de la Ley 1565 "Reforma Educativa” del 7 de julio de 1994, posteriormente la Ley 070 "Avelino Siñani Elizardo Pérez" de 20 de diciembre de 2010. Sin embargo, la resolución de problemas es implementada a partir de la tendencia curricular conocida como la matemática moderna "cuyas bases filosóficas de este movimiento se establecieron durante el seminario de Royamoundt, celebrado en 1959" (Álvarez, 2004, p. 45). Empero, investigadores como Capote en Pinar del Río Cuba, Ron en Cuba, Llivina en Cuba y Vargas en Mizque Bolivia puntualizan la persistencia de problemas en la enseñanza aprendizaje de la Matemática.

Capote (2003) sostiene que existen dificultades en la comprensión de los problemas 
que no permiten una adecuada búsqueda en la vía para la resolución, por su parte, Ron (2007) caracteriza el proceso enseñanza - aprendizaje ineficaz en la enseñanza de la resolución de problemas, mientras que Llivina (1999) concluye que el proceso de enseñanza aprendizaje es imperfecto a la hora de enseñar resolver problemas ya que se presentan bloqueos en el proceso de búsqueda de la vía de solución. En esta línea, también se advierte que el proceso de enseñanza aprendizaje de la Matemática no contempla la generación de un clima afectivo y emocional favorable para el aprendizaje, además, los momentos metodológicos: práctica, teoría, valoración y producción, correspondientes al MESCP y la Ley de Educación 070, se implementan sesgando el enfoque constructivista con la búsqueda de patrones que lleve a resolver problemas implementado operaciones de manera mecánica (Vargas, 2020).

Los problemas que se mencionan tienen raíces metodológicas, como factor común, ya que el proceso de enseñanza aprendizaje de la Matemática históricamente se ha caracterizado por tener un carácter mecánico de aplicación de fórmulas y procedimientos inalterables, hecho que no ha fortalecido la actividad mental y los procesos de pensamiento lógico, ello justifica la necesidad de indagar acerca de un procedimiento metodológico desarrollador de la resolución de problemas que permita romper los esquemas tradicionales en la enseñanza y aprendizaje de la Matemática.

Por esta razón, el presente estudio tiene como objetivo proponer una metodología de enseñanza aprendizaje de la Matemática orientada al desarrollo del pensamiento lógico matemático a partir de las dimensiones del aprendizaje desarrollador: activaciónregulación, significatividad y motivación. La misma está dirigida a los maestros del área de Matemática del nivel secundario de las unidades educativas del subsistema de educación regular del Estado Plurinacional de Bolivia.

Para ello, previamente es necesario puntualizar qué es el pensamiento lógico matemático y cual sus antecedentes. Hegel, en su Enciclopedia de las Ciencias Filosóficas (1830) ofrece la siguiente definición "La lógica es la ciencia de la idea pura; esto es, de la idea en el elemento abstracto del pensamiento. [...] su contenido no es otra cosa que nuestro pensamiento y sus ordinarias determinaciones" (Hegel, 1830, p. 87).

Por su parte, Spinoza, en su Tratado de la reforma del entendimiento (1988) el pensamiento lógico presenta propiedades esenciales de inteligencia o entendimiento donde sobresalen la certeza y la formalidad. Entre las características resalta el hecho de que percibe algunas cosas o forma ciertas ideas por sí mismo, y algunas a partir de otras; en cambio, las ideas del movimiento, no las forma, sino atendiendo a la idea de cantidad. De la misma manera, las ideas que forma expresan la infinitud $y$, las determinadas las forma a partir de otras. 0 simplemente, percibe las cosas, no en una acepción finita, si no bajo la noción de infinitud. Y, cuando imagina las cosas, las percibe en un número fijo y con determinada duración y cantidad. Así mismo, las ideas parecen derivarse de la sola necesidad de la naturaleza y poder humana, mientras que las ideas confusas se forman contra la voluntad. Las ideas que forman el entendimiento a partir de otras, la mente las puede determinar de muchas formas por ejemplo a través de la representación, las ideas son más perfectas cuando más perfección expresan de un objeto (Spinoza, 1988).

La cuestión de las relaciones entre la Lógica formal y los procesos de pensamiento atraviesa un momento de amplio debate en los medios científicos y filosóficos a partir de la segunda 
mitad del siglo XIX. En estos años comienza a configurarse la Psicología como ciencia empírica y experimental, mientras que, por otro lado, se producen notables desarrollos en la Lógica simbólica, empezando a ser considerada por algunos de sus cultivadores como una disciplina puramente deductiva y formal $\mathrm{y}$ tratando de eliminar (en ciertos casos) cualquier alusión a los procesos psicológicos reales de pensamiento. A partir de este momento podrán distinguirse, a grandes rasgos, dos posturas teóricas principales en lo referente a esta cuestión: la concepción psicologista y la logicista o platónica.

Para los que estudian la lógica desde una concepción psicologista, es coherente pensar que, efectivamente, las estructuras lógicas y matemáticas son asombrosamente exactas y objetivas, al margen del punto de vista subjetivo de quien las considere. No obstante, si estas entidades no se corresponden con ninguna propiedad perceptiva o física de los objetos, ni con ninguna construcción mental o representacional; en sus escritos sobre Filosofía de la Lógica, concretamente en El pensamiento: Una investigación lógica, Frege (1918) sostiene que: "El pensamiento no pertenece ni a mi mundo interior como representación, ni tampoco al mundo exterior, al mundo de las cosas perceptibles por los sentidos" (Frege, 1918, p. 221).

La llegada del siglo XX coincide con una nueva expansión y desarrollo de las investigaciones en Lógica y en Psicología experimental. Diversos lógicos de renombre optaron por la separación tajante entre las investigaciones lógicas y las psicológicas, siguiendo la línea de pensamiento de Frege. En este sentido, Russell (1904) opina que: "En cualquier parte de la Lógica y de las Matemáticas la existencia de la mente humana u otra es totalmente irrelevante; los procesos mentales son estudiados mediante la Lógica, pero el objeto de la Lógica no presupone procesos mentales" (Henle, 1962, p. 98).

En el siglo XX ha habido intentos de superar la dicotomía entre la postura psicologista (Boole) y la postura logicista (Frege o Husserl). Una de las principales aportaciones en esta línea la constituyen los trabajos de Piaget, que han tratado de establecer una Psicología y una Epistemología genéticas de corte naturalista y constructivista, en la que la actividad del sujeto desempeña un importante papel en los procesos de construcción del conocimiento científico y lógico - matemático. En los trabajos epistemológicos de Piaget (1970) hay numerosas menciones a la posición de la Lógica en el sistema general de las ciencias, e indicaciones de cómo surgen y se desarrollan en el curso evolutivo (filogenético y ontogenético) del sujeto que conoce.

Piaget (1969) considera que la Lógica y Psicología del pensamiento deben mantenerse separados en principio, pues la Lógica formal constituiría una axiomatización (o reconstrucción ideal) de los niveles más elevados a los que pueden llegar los procesos intelectuales, mientras que la Psicología del pensamiento sería una ciencia empírica que investiga cómo se piensa de hecho. Empero, Piaget (1969) establece que pueden existir conexiones entre las dos disciplinas, especialmente en el caso de los sujetos que disponen de la estructura de pensamiento conocida como operacional formal.

A partir de la teoría de Piaget se plantea como uno de los objetivos de la Matemática desarrollar el pensamiento lógico matemático, ya que se trata del área de conocimiento directamente relacionada con ella. Por esta razón, en los últimos años, diferentes investigadores, han coincidido que existe una necesidad imperante de estudiar el proceso de enseñanza aprendizaje de las Matemática debido a su impacto e importancia en el 
desarrollo del pensamiento lógico (Álvarez, 2004).

El proceso de enseñanza aprendizaje, a lo largo de la historia, ha sido parte implícita de las corrientes pedagógicas como el humanismo, cognitivismo (constructivismo y cognitivismo) que han tenido vigencia plena en momentos históricos, para luego ingresar a la decadencia y dar paso a otros que guardaban mayor relación con el momento histórico y cultural que se vivía (Álvarez, 2014).

El proceso de enseñanza aprendizaje de la Matemática ha incorporado metodológicamente la resolución de problemas como un elemento central en su desarrollo. Llivina (1999) caracteriza la resolución de problemas matemáticos como una capacidad específica que se desarrolla a través del proceso de enseñanza aprendizaje de la Matemática. Por su lado, Ron (2007) analiza el proceso de enseñanza aprendizaje de la resolución de problemas, desde el proceso de enseñanza aprendizaje desarrollador.

En este sentido la enseñanza aprendizaje desarrollador constituye una metodología que desarrolla el pensamiento en su concepción holística y holográfica (compleja). Castellanos (2001) define la enseñanza desarrolladora como el "Proceso sistémico de transmisión de la cultura en la institución escolar en función del encargo social, se organiza a partir de los niveles de desarrollo actual y potencial de los estudiantes y conduce el tránsito a niveles superiores de desarrollo" (Castellanos, 2001, p. 134). Estas dimensiones son activaciónregulación, significatividad y motivación.

La dimensión activación - regulación está referida a la actividad intelectual productiva creadora, es decir, no solo se trata de consumir y acumular información sino buscarla y producirla, problematizarla y usarla de manera consiente. Por su parte, la significatividad hace referencia a las relaciones significativas en el aprendizaje, en el área conceptual, experiencial y afectiva, es decir, la formación de sentimientos, actitudes y valores que son fundamentales en el siglo XXI. Y la motivación trabaja lo relacionado con las motivaciones intrínsecas hacia el aprendizaje, es decir, al sistema de autovaloraciones y expectativas positivas con respecto al aprendizaje.

En este sentido, la implementación de diferentes técnicas y estrategias en base a las dimensiones activación-regulación, significatividad y motivación hace posible desarrollar el pensamiento lógico matemático.

\section{MÉTODO}

El estudio se lleva adelante implementando el método empírico de la observación científica que permitió indagar acerca de las funciones del proceso de enseñanza aprendizaje a partir de sus componentes internos (problema, objeto, objetivo, contenido, método, medio, forma, resultado y evaluación) adoptado de Álvarez (2004). Trabajando de esta manera con docentes y estudiantes de segundo curso del nivel secundario constituyendo un estudio censal.

La dimensión teórica se trabaja con el método dialéctico y el método el hipotético deductivo. La dialéctica es adoptada como corriente teórica fundamental, permitió analizar a partir de la identificación de elementos y componentes que surgieron de estudios precedentes (contradicción, complementación y mutua necesidad). El método hipotético deductivo constituye una forma de abordaje transversal del tema de estudio, haciendo posible arribar a algunas conclusiones surgidas de una hipótesis, es decir, las mismas se alcanzan deductivamente.

La metodología que se elaboró para realizar la propuesta contempla el abordaje de la dimensión significatividad presente a la 
hora de resolver problemas matemáticos desde los procesos psíquicos que intervienen en el proceso de resolución de los problemas, por ser esta una actividad intelectual, "los procesos que intervienen en ella son predominantemente cognitivos" (Llivina, 1999, p. 52), complementados por la dimensión motivación que representa la intervención de procesos emocionales y volitivos constituyendo de esta manera "el pensamiento mismo, como proceso psíquico real, una unidad de lo intelectual y lo emocional" (Rubinstein, 1965, p. 208).

La dimensión activación - regulación contempla las capacidades intelectuales: memoria, imaginación, pensamiento y senso percepción están presentes en la resolución de problemas, haciendo referencia a las operaciones a través de las cuales el sujeto desarrolla la actividad intelectual (Rubinstein, 1995). La activación- regulación "expresa la caracterización cualitativa de las acciones intelectuales, por ende, de los procesos sobre cuya base transcurren" (Llivina, 1999, p. 54)

Las dimensiones dan pie a las seis etapas metodológicas cuyo mayor aporte es la integración de las acciones para resolver problemas y un proceso eslabonado de tareas. Han sido diseñados sobre la base las seis atapas de la función instructiva de la Teoría de los Procesos Consientes de Álvarez (2014).

\section{Primera etapa: Motivación y sentido}

La "motivación" en términos pedagógicos está relacionado con mostrar al estudiante aquello que no conoce y que se le quiere enseñar y al mismo tiempo hacerle entender que tiene la necesidad de aprender porque es muy importante. Está directamente relacionada con la primera acción para resolver problemas que se refiere a comprender el problema, para ello el sujeto tendrá que analizar, a partir de la lectura detallada del problema, separando lo dado de lo buscado, para lograr hallar alguna palabra clave $\mathrm{u}$ otro recurso que permita encontrar una adecuada orientación en el contexto de actuación.

Relacionar los elementos previamente analizados para expresar el problema con sus palabras o con un sistema simbólico abreviado o realizando una figura de análisis, construyendo una tabla o elaborando cualquier medio que sirva para modelar el texto. Para la realización de esta acción el sujeto deberá ejecutar operaciones propias del contexto matemático en el que está enunciado el problema.

\section{Segunda etapa o momento: Información del contenido en lenguaje lógico}

Se trata de un momento donde el docente explica a partir de un mapa de contenido el objeto o contenido, los conceptos, categorías, propiedades, magnitudes, clasificaciones, métodos de resolución de operaciones, resolución de casos de factorización, etc., de manera gráfica y sencilla, empleando un lenguaje apropiado.

Es un momento que vincula al estudiante con los conocimientos que le permitirán no solo resolver problemas de naturaleza didáctica, sino responder con herramientas científicas a algunos problemas de su comunidad o familia. Para una explicación más precisa, Condemarín (1996) propone:

La expresión verbal de un juicio lógico: negación, conjunción, disyunción y uso de cuantificadores, así mismo, la expresión simbólica de un juicio lógico: falso o verdadero, negación, conjunción y disyunción, así mismo, la noción de conservación: conservación de longitud, conservación de cantidad discontinua, conservación de cantidad continua, conservación de peso, conservación 
de número, Conservaciones de la equivalencia de dos conjuntos en correspondencia, Conservación de superficie, también la noción de seriación: seriación simple, seriación múltiple y la noción de clase: clasificación según una propiedad, clasificación simple y Noción de inclusión (Condemarín, 1996, p. 360-361).

\section{Tercera etapa o momento: La asimilación del contenido}

Es el momento de resolver problemas, inicialmente por parte del docente para que posteriormente también lo realicen los estudiantes sin perder de vista aquello que se había socializado en la etapa anterior ya que muchas de las interrogantes permitirán complementar el aprendizaje de los estudiantes.

Los procesos para resolver problemas puede comprender métodos como los propuestos por Polya en Álvarez (2004): que contempla comprender el problema, concebir un plan, ejecutar el plan y examinar la solución obtenida. Así mismo, el matemático Miguel de Guzmán plantea algunos métodos para la resolución de problemas, como la Resolución gráfica y Ensayo-error: cuyos pasos son: 1. Escoger una posible solución, 2. Efectuar las opresiones planteadas y probar si satisface al problema, 3. Se reajusta los procedimientos y se aplican las nuevas posibles soluciones hasta llegar a satisfacer el problema.

\section{Cuarta etapa o momento: La producción; puesta en práctica del contenido}

Se trata de una etapa o momento que presta atención a las inquietudes de los estudiantes que estén relacionadas con las actividades productivas y económicas propias de las familias y la comunidad. Presta atención a las cifras y los problemas de manera pormenorizada y a los métodos que se aplican.

El sujeto deberá ejecutar las operaciones de sintetizar al unificar los elementos separados en el análisis del problema para poder escribir la solución del mismo, considerando sólo aquellas propiedades que son necesarias o suficientes para la solución y aplicar, utilizando los elementos obtenidos en el análisis del problema en la solución del mismo.

\section{Quinta etapa o momento: La sistematización del nuevo contenido}

Constituye una etapa de organización a nivel de esquemas mentales los conocimientos nuevos a los ya existentes. Para ello será necesario relacionar elementos y componentes que surgieron en el proceso de resolución de problema, analizar el procedimiento implementado y sintetizar cada los procesos a partir de los elementos, componentes y algoritmos aplicados.

\section{Sexta etapa o momento: Evaluación del aprendizaje}

La evaluación es fundamental para el proceso, es así que se puede aplicar los diferentes tipos de evaluación. a) Según su finalidad y función: formativa y sumativa; b) según su extensión: global y parcial; c) según los agentes: interna (autoevaluación, heteroevaluación y coevaluación) y externa (evaluación de expertos); d) según el momento de aplicación: inicial, procesual y final. Se considera necesario enfocar el interés de la evaluación en el logro del objetivo, en la medida que se tenía planificado, debido a que la prioridad en la asimilación y acomodación o internalización de nuevos aprendizajes. 


\section{RESULTADOS}

Como se puede advertir el desarrollo del pensamiento lógico matemático no solo ha dejado de ser una prioridad sino que la misma no es trabajada desde los primero cursos en las Unidades Educativas del Sistema Educativo Plurinacional.

\section{Capacidades y habilidades lógicas según Piaget}

h. Clase de composición multiplicativa: se establecen relaciones entre varios conjuntos. Presentan diferentes variables que puede permitir se organice de diversas...

g. Clase de composición aditiva: donde se agrupa subclases parciales en una clase total, donde hay más elementos en el todo que en cada una de sus partes..

f. Una clase lógica es la unión de objetos con la misma cualidad en común. Supone que el niño comprende el conjunto de cualidades comunes de los objetos a ella.

e. Identifica patrones y secuencias aplicando algoritmos como la suma, resta, multiplicación, división, potenciación, etc.

d. Describe las relaciones secuenciales y no secuenciales sean de mayor a menor o viceversa (si $A$ es mayor que $\mathrm{B}$, y $\mathrm{B}$ mayor que $\mathrm{C}$, entonces $\mathrm{A}$ también es..

c. Aceptación de la conservación (reconoce la conservación bajo los argumentos de: identidad, donde una materia se mantiene sin añadir o quitar;...

b. Conservación momentánea (el niño reconoce la conservación solo en algunas circunstancias).

a. La no conservación: (debido a la influencia del factor perceptivo el niño considera que un material no conserva sus cualidades).

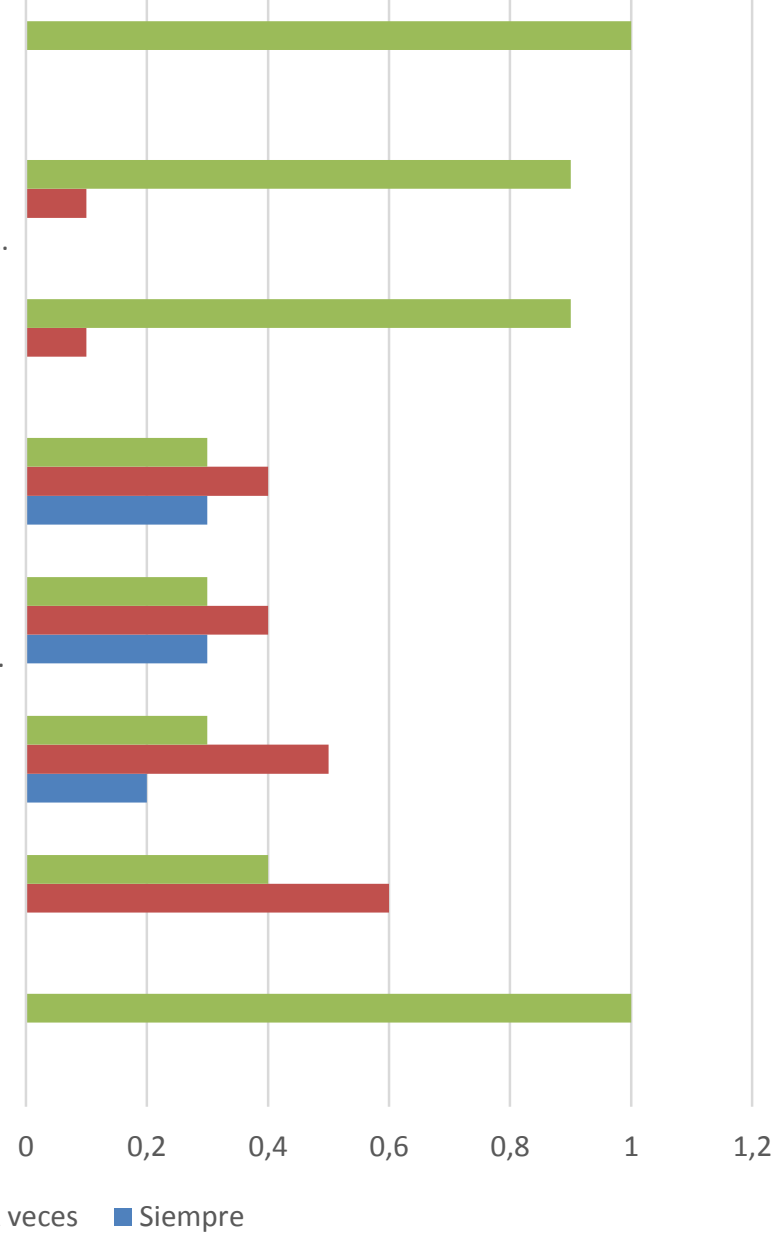

Nota: Para el cálculo de los porcentajes se utilizó una tabla de frecuencias

Las nociones no solo constituyen razonamientos fundamentales que están relacionadas con una determinada edad o periodo operatorio, en la Matemática es recurrentes su aplicación por ser parte constitutiva de un razonamiento para desarrollar un contenido. La no conservación (debido a la influencia del factor perceptivo el niño considera que un material no conserva sus cualidades). De todas las clases observadas el $100 \%$ de las veces no se emplea este razonamiento, peor aún de manera planificada.

La Conservación momentánea (el niño reconoce la conservación solo en algunas circunstancias). Al respecto se observa que el 
$60 \%$ de las veces, a veces se utiliza este razonamiento, sobre todo en algunas explicaciones del maestro, mientras que el $40 \%$ de las veces no se emplea aquello.

La aceptación de la conservación (reconoce la conservación bajo los argumentos de: identidad, donde una materia se mantiene sin añadir o quitar; reversibilidad, una materia retoma su estado inicial a pesar de haber sido modificada de forma). Los resultados revelan que el $20 \%$ de las veces se emplea este razonamiento, así mismo, el 50\% de las veces solo aparece esporádicamente y el $30 \%$ de las oportunidades no se lo emplea.

La "noción de seriación" que se hace evidente en las habilidades lógicas como: Describe las relaciones secuenciales y no secuenciales sean de mayor a menor 0 viceversa (si A es mayor que B, y B mayor que $C$, entonces $A$ también es mayor que $C$.). La habilidad es trabajada en un $30 \%$ de manera frecuente, es decir, siempre está presente, un $40 \%$ de las veces solo se presenta a veces y los otros $30 \%$ de las oportunidades no se hace referencia alguna. En lo que respecta a la identificación de patrones y secuencias aplicando algoritmos como la suma, resta, multiplicación, división, potenciación, etc., se aprecia que $30 \%$ de las veces se trabaja este razonamiento, pero el $40 \%$ de las oportunidades solo a veces y los otros $30 \%$ restantes no se advierte el uso y manejo del razonamiento.

La "noción de clase" es la tercera de las nociones que por alguna razón es la menos trabajada una clase lógica es la unión de objetos con la misma cualidad en común. Supone que el niño comprende el conjunto de cualidades comunes de los objetos a ella. Esta habilidad es trabajada el $10 \%$ de las veces esporádicamente, y el $90 \%$ de las veces no ocurre aquello. Otro razonamiento de clase es la denominada clase de composición aditiva: donde se agrupa subclases parciales en una clase total, donde hay más elementos en el todo que en cada una de sus partes $(A+B=C$, donde $\mathrm{A}$ es mayor que $\mathrm{B}$ ). Este razonamiento se evidenció el $10 \%$ de las veces de manera esporádica $\mathrm{y}$, los restantes $90 \%$ de las oportunidades no se trabaja dicha habilidad. Una tercera habilidad es la referida a la clase de composición multiplicativa: es aquella que busca se establezcan relaciones entre varios conjuntos. Presentan diferentes variables que puede permitir se organice de diversas formas.

El desarrollo de las capacidades y habilidades lógico matemáticos está relacionado con el logro de procesos lógicos, los mismos constituyen un elemento central del análisis de los procesos ejecutados. Al respecto se tiene los siguientes resultados. 


\section{Procesos lógicos relacionados con la matemática según Álvarez de Zayas}

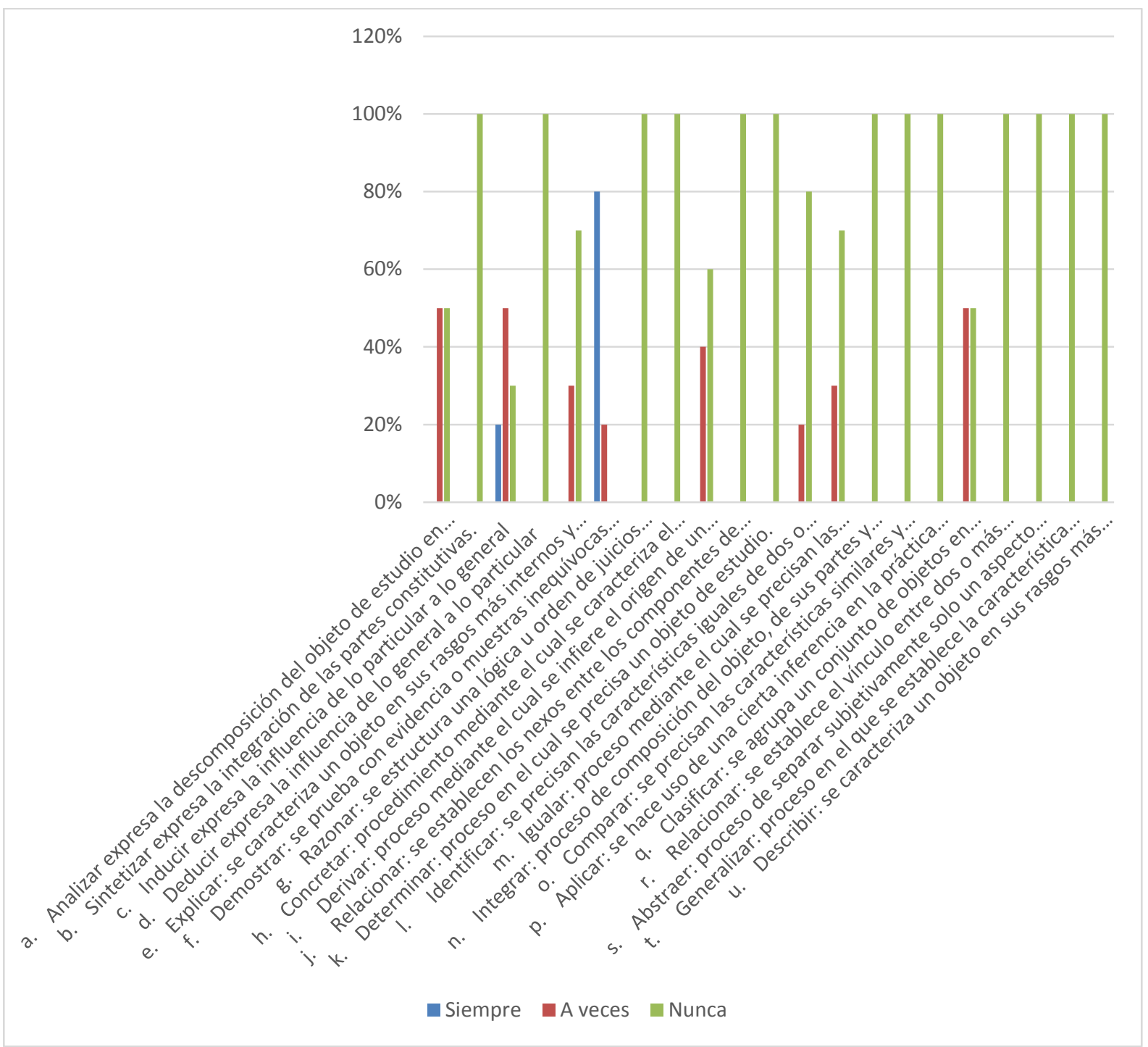

Nota: Para el cálculo de los porcentajes se utilizó una tabla de frecuencias

Se observa que los procesos lógicos que se desarrollan en la enseñanza-aprendizaje la Matemática son reducidos, como se advierte: $E l$ Análisis que expresa la descomposición del objeto de estudio en sus partes, sobre el mismo, según lo observado se advierte: siempre el $0 \%$, a veces $50 \%$ y nunca $50 \%$.

Sintetizar: expresa la integración de las partes constitutivas. Se advierte: siempre $0 \%$, a veces $0 \%$ y nunca el $100 \%$.

Inducir: expresa la influencia de lo particular a lo general. Al respecto: siempre $20 \%$, a veces $50 \%$, nunca $30 \%$.

Deducir: expresa la influencia de lo general a lo particular. Se evidencia que: siempre $0 \%$, a veces $0 \%$ y nunca $100 \%$.

Explicar: se caracteriza un objeto en sus rasgos más internos y esenciales, los resultados: siempre el $0 \%$, a veces $30 \%$ y el $70 \%$ nunca. La explicación lo realiza el docente abarcando aspectos procedimentales para la resolución de las operaciones. 
Demostrar: se prueba con evidencia o muestras inequívocas la verdad de una proposición, al respecto se observó que: Siempre $80 \%$, a veces $20 \%$, nunca $0 \%$. La demostración es parte esencial de la enseñanza de la matemática, sin embargo, al no estar planificado este proceso se limita solo al maestro.

Razonar: se estructura una lógica u orden de juicios encaminado a demostrar algo. Se observa: Siempre $0 \%$, casi siempre $0 \%$ y nunca el $100 \%$. En el proceso de resolución de problemas solo se aprende a resolver operaciones.

Concretar: procedimiento mediante el cual se caracteriza el objeto en sí mismo. Se advierte: siempre $0 \%$, casi siempre $0 \%$ y nunca $100 \%$. No se aplica el proceso lógico.

Derivar: proceso mediante el cual se infiere el origen de un objeto. El 0\% siempre, $40 \%$ a veces y el $60 \%$ nunca. Se evidencia que se emplea al derivar algunas operaciones de otras.

Relacionar: se establecen los nexos entre los componentes de un objeto o entre objetos. Se observa: siempre $0 \%$, casi siempre $0 \%$ y nunca el $100 \%$. No se alcanza procesos de este nivel.

Determinar: proceso en el cual se precisa un objeto de estudio. Al respecto se observa: $0 \%$ siempre, $0 \%$ casi siempre y $100 \%$ nunca, ya sea de manera práctica o teórica.

Identificar: se precisan las características iguales de dos o más objetos de estudio. Al respecto: siempre $0 \%$, casi siempre $20 \%$ y $80 \%$ nunca. Se lo emplea para reconocer el tipo de operación Matemática.

Igualar: proceso mediante el cual se precisan las características iguales de dos o más objetos de estudio. Se observó su implementación con los siguientes resultados: siempre $0 \%$, casi siempre $30 \%$, y nunca $70 \%$. Se lo emplea como parte del lenguaje matemático sin hacer referencia directa a procesos lógicos.

Integrar: proceso de composición del objeto, de sus partes y sus características. Los resultados reflejan una aplicabilidad: siempre $0 \%$, casi siempre $0 \%$ y nunca $100 \%$.

Comparar: se precisan las características similares y diferencias entre dos objetos de estudio. Se advierte: siempre 0\%, casi siempre $0 \%$ y nunca $100 \%$. No se emplea el proceso lógico.

Aplicar: se hace uso de una cierta inferencia en la práctica para transformar una situación. Al respecto se observó: siempre $0 \%$, casi siempre $0 \%$ y nunca $100 \%$. Se emplea el concepto para utilizar un algoritmo y no como proceso lógico útil en el procedimiento.

Clasificar: se agrupa un conjunto de objetos en correspondencia con cierta característica. Se evidencia los siguientes resultados: siempre 0\%, casi siempre $50 \%$ y nunca $50 \%$. Se lo maneja como adjetivo para algunas tareas.

Relacionar: se establece el vínculo entre dos o más características de un objeto de estudio o con otros. Los resultados muestran su uso: siempre $0 \%$, casi siempre $0 \%$ y nunca $100 \%$. No se emplea el proceso lógico.

Abstraer: proceso de separar subjetivamente solo un aspecto del objeto. La aplicación de este proceso lógico arroja lo siguiente: siempre $0 \%$, casi siempre $0 \%$ y nunca $100 \%$. No se emplea el proceso lógico.

Generalizar: proceso en el que se establece la característica común de dos o más objetos. La observación muestra: siempre $0 \%$, casi siempre $0 \%$ y nunca $100 \%$, no se emplea el proceso lógico.

Como se puede advertir la enseñanza aprendizaje de la Matemática no está orientada al desarrollo del pensamiento lógico matemático, por lo tanto, se plantea la siguiente: 


\section{Propuesta metodológica}

La misma permite concretar en tres fases las actividades que se deben llevar adelante, involucrando las dimensiones del aprendizaje desarrollador, las etapas o momentos metodológicos y los procesos lógicos concordantes con cada una de ellas. Se inicia con una prueba de carácter proposicional del diagnóstico

\section{Fase I: Prueba Objetiva de Diagnóstico}

¡Lee con cuidado todos los ejercicios antes de empezar a resolver!

1. Un día Juan se encuentra ante un cierto número de objetos diversos como ser vasos de plástico, piezas de madera, platos de porcelana, una esponja para lavar platos, cucharas de madera, plástico y metal, un carrito de plástico y varias pelotitas de plástico. Todas ellas son colocadas en un recipiente con agua. A partir de lo que va ocurriendo con cada uno de las piezas debes clasificar en grupos ¿qué grupos podría surgir y por qué?

Anota en los recuadros los objetos que correspondan a un grupo (ponga un nombre a cada grupo), puedes añadir más recuadros en caso de ser necesario:

2. ¿Por qué algunos objetos flotan y otros no?

$\mathrm{R}:$

Resuelve las siguientes operaciones:

3. Las longitudes de los lados de un triángulo son 19mm, 32mm, 13mm. Determine el perímetro del triángulo.

$\mathrm{R}:$

4. Las rectas ly m son paralelas. ¿Cuál es la amplitud del ángulo x?

$\mathrm{R}:$

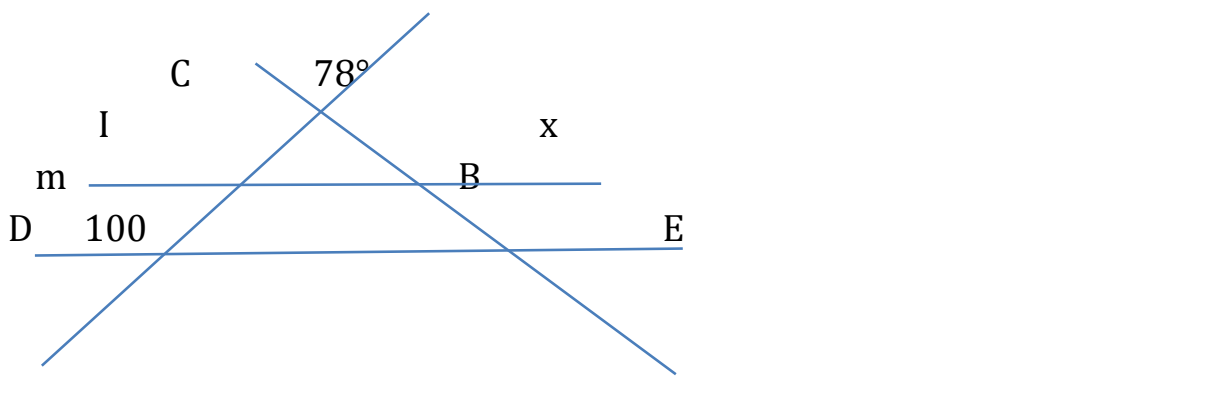

5. Las longitudes de los lados de un triángulo se expresan como $\mathrm{x}+1,7-\mathrm{x}, 4 \mathrm{x}-2$.

Determina todos los valores que puede tomar $\mathrm{x}$ para que el triángulo sea isósceles. $\mathrm{R}$ :

6. En la figura $\mathrm{MN} \overline{\mathrm{ll}} \mathrm{PQ}, \overline{\mathrm{RN}}$ bisectriz del ángulo SRQ y $\theta=20$.

Clasifique el triángulo PQR según la longitud de sus lados y según la amplitud.de sus ángulos. 


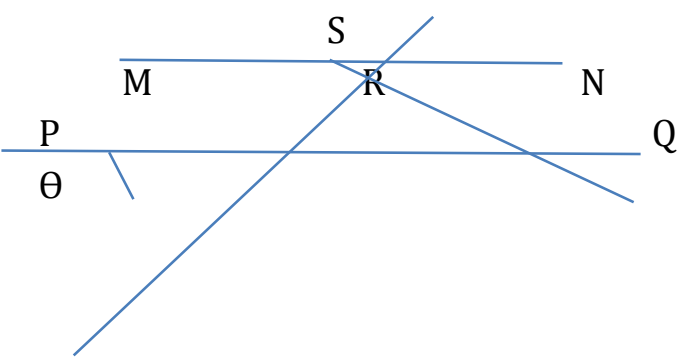

$\mathrm{R}:$

Los ejercicios planteados presentan los siguientes criterios: Un ejercicio sin solución, un ejercicio con varias vías de solución y un ejercicio que vincula la geometría con el trabajo con variables. Esto se trabajó con la finalidad de analizar los aciertos, los errores, pero por sobre todo la utilización de diferentes vías de solución que constituyen el reflejo de la capacidad específica a la hora de resolver problemas.

En este sentido, los resultados del diagnóstico proporcionan elementos que permiten tomar decisiones acerca de la metodología, los contenidos, los objetivos y las metas. Dicho proceso de análisis se lo realiza sobre la base de dimensiones e indicadores siguientes:

\section{a. Dimensión Motivación:}

\begin{tabular}{lcccc}
\hline Indicadores & $\begin{array}{c}\text { Muy } \\
\text { buena (4) }\end{array}$ & $\begin{array}{c}\text { Buena } \\
\text { (3) }\end{array}$ & $\begin{array}{c}\text { Regular } \\
\text { (2) }\end{array}$ & $\begin{array}{c}\text { Mala } \\
\text { (1) }\end{array}$ \\
\hline 1. Interés personal por aprender matemática. & & & & \\
2. Interés personal por resolver problemas. & & & & \\
3. Presenta la seguridad necesaria para esforzarse. & & & & \\
4. Persevera a pesar de los obstáculos que puedan surgir. & & & & \\
\hline
\end{tabular}

\section{b. Dimensión Activación- regulación}

\begin{tabular}{|c|c|c|c|c|}
\hline Indicadores & Muy buena (4) & Buena (3) & Regular (2) & Mala (1) \\
\hline \multicolumn{5}{|l|}{ 1. Independencia. } \\
\hline \multicolumn{5}{|l|}{ 2. Originalidad. } \\
\hline \multicolumn{5}{|l|}{ 3. Fluidez. } \\
\hline \multicolumn{5}{|l|}{ 4. Flexibilidad. } \\
\hline \multicolumn{5}{|l|}{ 5. Logicidad. } \\
\hline \multicolumn{5}{|l|}{ 6. Profundidad. } \\
\hline 7. Organización. & & & & \\
\hline
\end{tabular}


c. Dimensión: Significatividad

\begin{tabular}{lcccc}
\hline \multicolumn{1}{c}{ Indicadores } & $\begin{array}{c}\text { Muy } \\
\text { buena (4) }\end{array}$ & $\begin{array}{c}\text { Buena } \\
\text { (3) }\end{array}$ & $\begin{array}{c}\text { Regular } \\
\text { (2) }\end{array}$ & $\begin{array}{c}\text { Mala } \\
\text { (1) }\end{array}$ \\
\hline 1. Significatividad conceptual. & & & & \\
2. Significatividad experiencial. & & & \\
3. Significatividad afectiva. & & & \\
4. Analiza las consecuencias de los contenidos & & & \\
5. Solidaridad. & & & \\
6. Responsabilidad. & & & \\
\hline
\end{tabular}

En los cuadros se desglosan las dimensiones en indicadores que son calificadas con criterios: Muy Bueno (4), Bueno (3), Regular (2), Malo (1); una vez centralizado permite apreciar los niveles de desarrollo de las diferentes dimensiones.

\section{Fase II de Implementación: Ejecución de las acciones del aprendizaje desarrollador y las etapas metodológicos}

Las diferentes dimensiones serán trabajadas tomando en cuenta tres momentos: el primer momento es la explicación de las consignas de trabajo. El segundo momento será trabajada de manera individual o grupal aplicando métodos heurísticos y, por último, se implementará el momento de la evaluación que servirá para analizar los aciertos y desaciertos que se hayan tenido al momento de resolver los problemas.

Para llevar adelante la propuesta metodológica se debe adoptar las siguientes medidas:

- La cuidadosa preparación de los ejercicios y problemas a utilizar en las clases; así como los métodos de enseñanza y las formas en que presentan los ejercicios y problemas.

- La participación activa y motivada de los estudiantes.

Para desarrollar el pensamiento lógico matemático se debe potenciar las dimensiones del aprendizaje desarrollador como ser: a. activación - regulación, b. significatividad, y c. Motivación. En este sentido, se presentan los problemas adecuadas dirigida a cada una de las dimensiones, las mismas que son reforzadas con las etapas o momentos metodológicos.

\section{Dimensión Motivación}

Se puede trabajar ejercicios y problemas donde se utilicen: cuadrados mágicos, varillas de igual longitud, soluciones novedosas, contar, como vía de solución.

1. En un cuadrado mágico la suma de las casillas horizontal, vertical y diagonal es siempre la misma. En el siguiente cuadrado mágico $\mathrm{A}+\mathrm{B}+\mathrm{C}=-34,5$. Halla $\mathrm{A}, \mathrm{B}, \mathrm{C}, \mathrm{D}$ y $\mathrm{E}$

R: $D=-6,9 ; E=-16,1 ; \quad A=-2,3 ; C=-20,7 ; \quad B=-11,5$ (Cuadrados mágicos)

\begin{tabular}{|c|c|c|}
\hline$-18,4$ & $A$ & $-13,8$ \\
\hline$D$ & $B$ & $E$ \\
\hline$-9,2$ & $C$ & $-4,6$ \\
\hline
\end{tabular}


2. Se pretende dividir el tronco cilíndrico de la figura en 8 trozos iguales, pero solamente con tres cortes. ¿Cómo serían esos cortes?

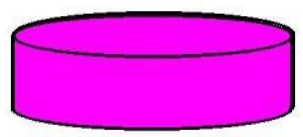

R:

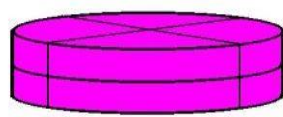

(Soluciones novedosas)

3. Diga cuántos rectángulos hay en la siguiente figura.

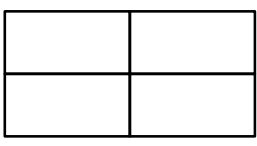

R: 9. (Conteo, establecer un orden)

4. Si en un momento determinado adelanta usted al segundo, ¿en qué lugar se colocaría?

R: en segundo (Búsqueda de alternativas creativas)

\section{Dimensión Activación-regulación}

Para ejecutar las acciones orientadas a la dimensión activación-regulación, se toman en cuenta los siguientes tipos de ejercicios:

\section{Ejercicios con solución única:}

1. Construya un rombo en el que una de sus diagonales sea igual a uno de sus lados.

R: A partir de la utilización de la amplitud de los ángulos de un triángulo equilátero, se llega a que son, $60^{\circ}, 60^{\circ}, 120^{\circ}, 120^{\circ}$. (Estrategia: graficar, analizar casos particulares, establecer relaciones, buscar regularidades).

2. En una selva hay algunas hienas, águilas y serpientes. Cada mañana, cada una de las hienas se come un águila. En la tarde cada serpiente se come una hiena. Por la noche cada águila se come una serpiente. Al final del tercer día solo queda un águila. ¿Cuántos animales de cada tipo había al principio?

R: Hienas 13, Águilas 19, Serpientes 9 (Estrategia: trabajo hacia atrás)

Orientaciones al profesor: promueve la utilización de estrategias generales como: tanteo inteligente, hacer un diagrama, usar las propiedades, resolver un ejercicio equivalente, trabajar hacia atrás, reformular el problema, tomar un descanso y después intentarlo y promover que se valore la utilidad de la estrategia usada, y bajo qué condiciones se puede volver a utilizar.

\section{Ejercicios con más de una solución:}

1. Si la siguiente pieza representa un cuarto de otra.

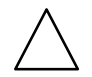

Representa más de tres piezas enteras con la condición de que al menos uno de sus lados descanse sobre una línea horizontal imaginaria.

R:
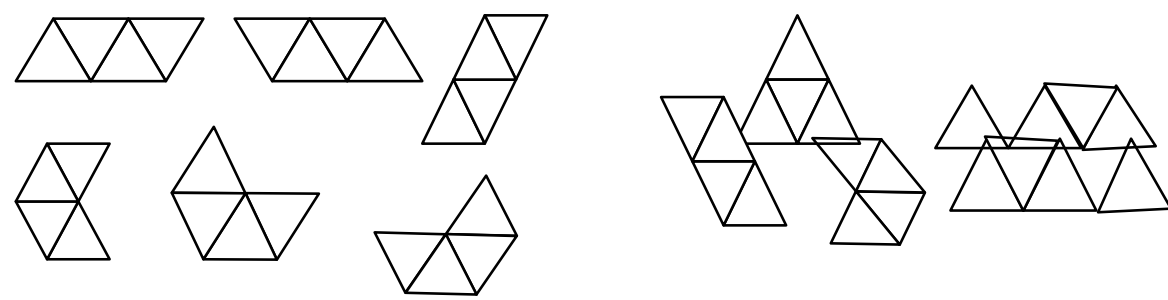
2. Determinar operaciones combinadas de aritmética cuyo resultado final sea 13 . Incluir las 4 operaciones elementales $(+,-, \cdot,:)$ al menos una vez, o alternativamente, exactamente una vez.

R: $((22+5) \cdot 2-1): 4 ;($ Infinitas soluciones)

3. Todos los dígitos escondidos en la adición son impares diferentes.

Reconstruye la adición.

\begin{tabular}{|c|c|c|c|}
\hline R: 19 & 37 & 17 & 39 \\
\hline+37 & +19 & +39 & +17 \\
\hline & 56 & 56 & \\
\hline
\end{tabular}
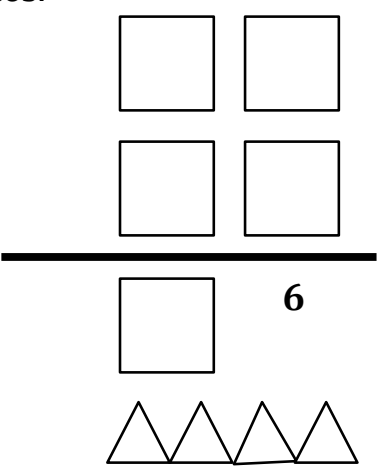

Orientaciones al profesor: Dejar que los estudiantes intenten resolver el ejercicio sin importar la vía que utilicen, valorar cada una de las vías y soluciones encontradas, y exigir la búsqueda de otras, pedir la fundamentación de otras soluciones encontradas, trabajar con los datos para obtener situaciones y soluciones diferentes que incluso pueden ser hasta únicas y sin solución.

\section{Ejercicios sin solución:}

1. ¿Cuál es el perímetro del triángulo de lados $94 \mathrm{~mm}, 177 \mathrm{~mm}$ y $83 \mathrm{~mm}$ ?

R: N.S. No existe el triángulo (Datos contradictorios)

2. ¿Cuáles pueden ser las dimensiones de un cuadrado, si se sabe que la longitud de sus lados son cantidades enteras, su área es menor que $160 \mathrm{~cm}^{2}$ y el número que representa su área termina en dos?

R: N.S. (ningún cuadrado perfecto termina en dos)

3. La suma de tres números pares consecutivos es 57. ¿Cuáles son esos números?

R: N.S. (La suma de tres números pares siempre es par)

Orientaciones al profesor. Dejar que los estudiantes resuelvan el ejercicio, sin importar que no encuentren que no tiene solución. Si el estudiante da alguna respuesta como solución: Que explique cómo llegó a la solución, buscar contraejemplos, y cuestionar la solución hasta que el estudiante identifique que ese ejercicio no tiene solución.

Que el estudiante explique por qué no fue capaz de llegar a que no tiene solución.

Que el estudiante reformule el problema para que tenga solución, que puede ser hasta más de una. 


\section{Ejercicios contra el tiempo:}

1. Calcula:
a) $36-25=R: 11$
b) $(50-49)+(49-48)+(48-47)++(2-1)=R: 49$
c) $2-(1-(2-(1-(2-(1-2)))))=$
R: 5

2. Ana dibuja todos los triángulos de lados enteros y perímetro 9. Patricia dibuja todos los triángulos de lados enteros y perímetro 10. ¿Cuál de las dos dibuja más triángulos?

R: Ana 3, Patricia 2. Ana dibuja más triángulos.

Orientaciones al profesor: Determinar un tiempo para dar la solución.

Al concluir el tiempo discutir las vías desarrolladas, las soluciones encontradas, si no se encontraron, sugerir, intercambiar, nunca resolverlo, si se encontraron, reconocer el trabajo desarrollado.

\section{Dimensión Significatividad}

Para la puesta en práctica de la dimensión significatividad se plantean los siguientes ejercicios:

\section{Ejercicios con omisión de condiciones o preguntas, o adición de información no relevante para el proceso de solución:}

1. La figura $\mathrm{ABCD}$ es un rectángulo de área $24 \mathrm{~cm}^{2}$.

E es el punto medio de $A B$.

¿Qué porciento representa el área sombreada

A

$\mathrm{D}$

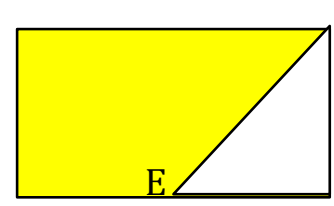

$\mathrm{C}$

B

del área del rectángulo?

R: 75\% (Por diferencia de áreas, proporcionalidad, casos particulares)

2. Esteban ya ganó 50 de 75 juegos contra su computadora. Si quiere sostener en total 105 juegos. ¿Cuántos juegos más tiene que ganar para que al final de la jornada le haya ganado a su computadora el $60 \%$ de los juegos?

R: 13 juegos

3. Si el valor de A es el $30 \%$ del de B, el valor de B es el $40 \%$ del de $\mathrm{C}$ y el valor de $\mathrm{D}$ es el $60 \%$ del de C. Calcula A/B

R: $3 / 10$ 
Orientaciones al profesor. No adelantar información al estudiante, dejar que éste intente resolverlo con sus propios conocimientos. Si los estudiantes no utilizan la información no relevante, se pedirá que justifiquen su no utilización, y se orientará la elaboración y resolución de un nuevo problema sin la información no relevante.

Si los estudiantes utilizan la información no relevante, se orientará resolverlos sin esta información y comparar las soluciones hasta que se identifique la no necesidad de la información para el proceso de resolución. De esta manera, se alcanzará la sistematicidad.

\section{Fase III: Evaluación del desarrollo de las dimensiones}

El cuestionario será auto aplicado ya que se pretende llevar adelante un proceso de autoevaluación orientada a reflexionar el proceso y las operaciones implementado en la búsqueda de desarrollar las dimensiones del aprendizaje desarrollador por su intermedio el pensamiento lógico matemático. Posteriormente se sugiere evaluar los indicadores que posiblemente no han sido lo suficientemente trabajadas.

\section{Discusión}

Para Koliaguin (1975) el pensamiento matemático se caracteriza por la profundidad, la amplitud, el carácter autocrítico del pensamiento y la flexibilidad. Mientras que Rodríguez (2003) considera al pensamiento lógico matemático es una capacidad que permite interpretar información en la vida diaria, tomar decisiones en función de esa interpretación, usar herramientas matemáticas incluyendo la modelación, el pensamiento analítico, crítico y flexible. Adoptando ambas definiciones se advierte que es amplio y no se cierra a una actividad concreta, sin embargo, sus características la hace distinta a otras habilidades del pensamiento (Spinoza, 1988).

A partir de los estudios y el método de Polya (1973) acerca de la enseñanza de la Matemática donde los contenidos pasan a un segundo plano frente a la prioridad de desarrollar el pensamiento lógico matemático surgen diversas propuestas fruto de investigaciones y estudios en el campo de la Didáctica de la Matemática, como la de Müller (1978), Schoenfeld (1985) y Santos (1993).

Müller (1978) desarrolla lo que se denomina instrucción heurística, que se resume en procedimientos para facilitar la búsqueda de la vía de solución, constituyen un sistema que abarca: 1. Orientación hacia el problema; 2. Trabajo en el problema; 3. Solución del problema y 4. Evaluación de la solución y la vía. Por un lado, Schoenfeld (1985), integra la resolución de problemas con el desarrollo del pensamiento lógico matemático, y cuyo método comprende: 1. Comprensión del problema; 2. Diseño de un plan de solución; 3. Ejecutar el plan y 4. Mirada retrospectiva. Y por otro lado, Santos (1993) modela el proceso de resolución en ocho fases, inspirados en el desarrollo de una investigación científica: 1. Consciencia de la existencia del problema; 2. Supresión de los dados; 3. Interés por la situación problemática abordada; 4. Análisis cualitativo; 5. Formulación de hipótesis; 6. Estrategias de resolución; 7. Análisis de los resultados y 8. Maduración.

Los métodos propuestos por Müller (1978), Schoenfeld (1985) y Santos (1993) comprenden diferentes procesos lógicos de carácter sistémico. Entre sus coincidencias se puede advertir que parten de la comprensión o la conciencia de que existe un problema, para luego plantear o buscar una solución que puede ser deducido, inferido o hipotetizado, la resolución pasa por un momento de planificación y ejecución que será desarrollada 
a partir de los datos y los conocimientos previos que tenga el individuo $y$, finalmente culminar con una evaluación del resultado que permitirá verificar si los datos alcanzados satisfacen al problema, en caso de no ser así, será necesario replantear y ejecutar nuevamente el procedimiento. El proceso la resolución de problemas no solo pasa por la ejecución de una o varias operaciones matemáticas, sino también, el razonamiento lógico. Sin embargo, los autores no prestan mucha atención al desarrollo sistemático de las operaciones lógicas (ej. Analizar, deducir, caracterizar, comparar) que son fundamentales a la hora de solucionar un problema sean cuantitativos o cualitativos (Álvarez, 2004).

De la misma forma, la resolución de problemas a girado en torno al contenido, siendo este el componente central del proceso de enseñanza aprendizaje de la Matemática, ya que la resolución de problemas se utiliza como el mecanismo para enseñar un contenido, situación que no permite desarrollar el pensamiento lógico matemático como prioridad. A pesar que existe una estrecha relación entre la resolución de problemas y el desarrollo del pensamiento lógico matemático, ya que se incita al estudiante a implementar procesos característicos del pensamiento lógico (Silvestre, 2001).

En el presente estudio, el autor pudo advertir que los maestros sostienen y enseñan empleando la resolución de problemas sin emplear un método concreto correspondiente a un autor, lo que ha generado un sesgo no solo en lo metodológico sino también en el enfoque constructivista, que aun predomina como enfoque pedagógico. Dicho sesgo se traduce en la identificación de uno o dos operaciones matemáticas para luego aplicar mecánicamente y arribar a la solución. Por lo tanto, no se desarrolla el pensamiento lógico, ya que la repetición y mecanización no facilitan aquello.
Castellanos (2001) considera que el desarrollo del pensamiento matemático no pasa solo por el trabajo sistemático de las habilidades y capacidades directamente relacionadas a ella, a criterio de la autora es necesario trabajar de manera integral y holística las dimensiones activación-regulación, significatividad y motivación. Las mimas abarcan el desarrollo no solo del pensamiento sino también del lenguaje, ya que ambos se necesitan, complementan a pesar de ser contrarios (relación dialéctica), los valores humanos y espirituales también son parte del desarrollo del pensamiento lógico matemático. El autor se adscribe al planteamiento de Castellanos (2001) comprendiendo que el pensamiento lógico matemático involucra el desarrollo de otras habilidades y capacidades como ser el lenguaje, los valores humanos y espirituales que en la vida cotidiana no están desvinculados unos con otros.

A las dimensiones del aprendizaje desarrollador se integran las fases o momentos de la función instructiva del proceso de enseñanza aprendizaje de Álvarez (2004). Las dimensiones: motivación, activación-regulación y significatividad guardan relación directa con las etapas: motivación, información del contenido, asimilación del contenido, dominio del contenido, sistematización del nuevo contenido y la evaluación del aprendizaje (Álvarez, 2004). Dando paso a las etapas: motivación y sentido, información del contenido en lenguaje lógico, asimilación del contenido, producción puesta en práctica del contenido, sistematización del nuevo contenido y, evaluación del aprendizaje.

La relación entre las dimensiones del aprendizaje desarrollador y las etapas metodológicas, así como su relación con los componentes del proceso de enseñanza aprendizaje se presenta en el siguiente cuadro: 


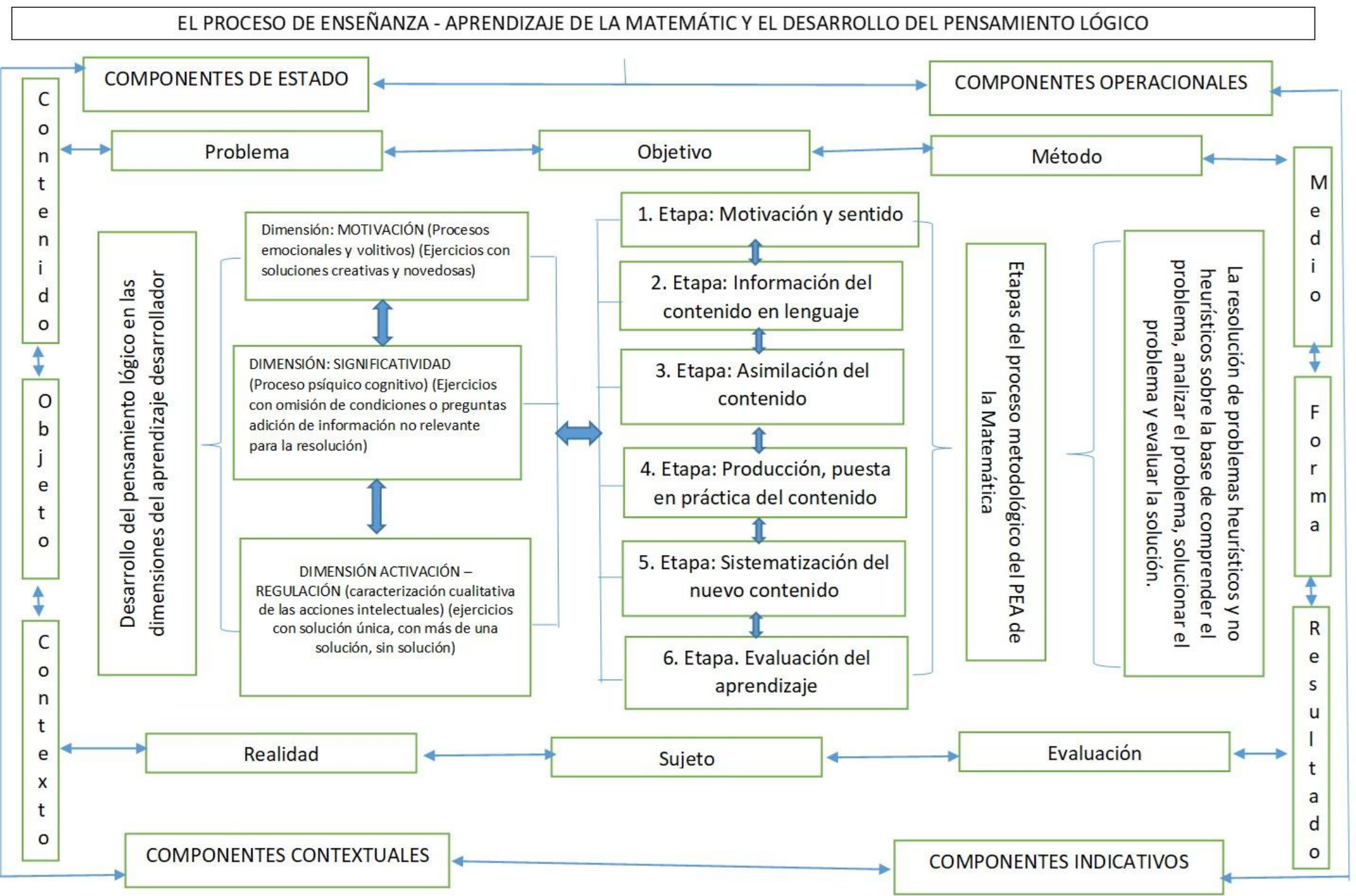




\section{CONCLUSIONES}

El estudiante no debe ser concebido como un sujeto que sigue un conjunto de pasos para resolver problemas, sino como aquel que moviliza y desarrolla su pensamiento matemático en la búsqueda de vías de solución a diferentes problemas que se le presenten.

El desarrollo del pensamiento lógico matemático es y será una de las prioridades de la educación impartida por las Unidades Educativas del sistema regular, su alta importancia radica en que la tecnología y su lenguaje algebraico, el sistema binario y otros contenidos y la habilidad de manejarlo de manera creativa es y será aún más fundamental con el mayor avance de la ciencia y la tecnología.

La propuesta metodológica para contribuir al desarrollo de la capacidad para resolver problemas matemáticos fortalece las dimensiones del aprendizaje desarrollador: Motivación, activación-regulación, significatividad con conceptos y aportes del enfoque cognitivista, histórico social y dialectico, holístico holográfico. Está concebida para desarrollarse en seis fases o momentos, subordinadas a las dimensiones: motivación, activación-regulación, significatividad en el proceso de enseñanza - aprendizaje de la resolución de problemas matemáticos y de manera indirecta se desarrolla el pensamiento lógico matemático.

REFERENCIAS

Álvarez, E y Álvarez de Zayas, C. (2004) Elementos Epistemológicos de la Matemática y su enseñanza. Bolivia: Kipus Álvarez, C. (2014) Didactica Elemental. Bolivia: Kipus

Capote, M. (2003) Una estructuración didáctica para la etapa de orientación en la solución de problemas aritméticos con texto en el primer ciclo de la escuela primaria. Tesis Doctorado en Ciencias Pedagógicas Universidad "Hermanos Saiz Montes de Oca", Pinar del Río

Castellanos, D. (2001) Hacia una concepción del aprendizaje desarrollador. 1. ed. La Habana: Instituto Superior Pedagógico "Enrique José Varona"

Condemarín, M. (1989) Madurez Escolar. Ediciones UC. Santiago de Chile

Frege, G. (1918) Los fundamentos de la aritmética. Madrid

Hegel, G. W. F. (1830) Enciclopedia de las Ciencias Filosóficas. Traducción española de E. Ovejero y Maury México: Editorial Porrúa, 1997

Henle, M. (1962): "Sobre la relación entre la Lógica y el Pensamiento". En: J. Delval (Compilador): Investigaciones sobre lógica y psicología. Madrid: Alianza, 1977

Inhelder y Piaget (1985) De la lógica del niño a la lógica del adolescente: Ensayo sobre la construcción de las estructuras operatorias formales. (Cevasco, traductor). Paidos Barcelona

Koliaguin, Y. M. (1975) Metodología de la enseñanza de la Matemática en la escuela media. 1. ed. Moscú: Editorial Instrucción

Llivina, M. (1999) ¿Modelos para resolver problemas? En Revista de Educación Matemática. Departamento de Matemáticas. Universidad Tecnológica de Pereira. Colombia. Número 3

Müller, H. (1978) El trabajo heurístico y la ejercitación en la enseñanza de la Matemática en la enseñanza general, politécnica y laboral. - Instituto Superior Pedagógico "Frank País García", Santiago de Cuba

Piaget, J. (1969) La Construcción de lo real en el niño. Edición Revolucionaria, La Habana, Cuba

Polya, G. (1973) How solve it. A New Aspect of Mathematical Method. 2. ed. New Jersey: Princeton University Press

Rodríguez, J. B. (2003) Una Propuesta Metodológica para la utilización de las tecnologías de la información y las comunicaciones en el proceso de 
enseñanza-aprendizaje de las funciones matemáticas. Tesis Doctorado Instituto Superior Pedagógico "Enrique José Varona", La Habana

Ron, J. (2007) Una Estrategia Didáctica para el proceso de enseñanza-aprendizaje de la Resolución de Problemas en las clases de Matemática en la educación Secundaria Básica. Tesis Doctorado. Instituto Superior Pedagógico "Enrique José Varona", La Habana

Santos, M. S. (1993) A metodologia de resolução de problemas como atividade de investigação: um instrumento de mudança didática. Tese (Doutorado em Educaçao) - Faculdade de Educação, Universidade de São Paulo, São Paulo

Schoenfeld, A. H. (1992) Learning to think mathematically: problem solving, metacognition, and sense-making in mathematics. En: GROUWS, D. (Ed.). The Handbook for Research on Mathematics Teaching and Learning. New York: Mac Millan

Silvestre, M. (2001) Aprendizaje, educación y desarrollo. 1. ed. La Habana: Editorial Pueblo y Educación. Universität de Valencia

Spinoza, B. (1988). Tratado de la reforma del entendimiento. s/e.

Vargas, W. (2020) Metodología de la enseñanza aprendizaje de la matemática orientada al desarrollo del pensamiento lógico matemático para estudiantes de Segundo de secundaria. Tesis Doctoral. Universidad Autónoma del Beni 